\title{
Municipios indígenas de Guatemala: conocimientos, actitudes y prácticas frente a la COVID-19 resultados de la segunda ronda de recopilación de datos
}

Population Council

Follow this and additional works at: https://knowledgecommons.popcouncil.org/departments_sbsr-pgy

Part of the International Public Health Commons, Public Health Education and Promotion Commons, and the Social and Behavioral Sciences Commons

How does access to this work benefit you? Let us know!

\section{Recommended Citation}

"Municipios indígenas de Guatemala: conocimientos, actitudes y prácticas frente a la COVID-19 resultados de la segunda ronda de recopilación de datos," presentation, COVID-19 Research \& Evaluations. Guatemala: Population Council, 2020. 


\section{POPULATION}

\section{COUNCIL}

Ideas. Evidencia. Impacto.

MUNICIPIOS INDÍGENAS DE

GUATEMALA: CONOCIMIENTOS,

ACTITUDES Y PRÁCTICAS FRENTE

A LA COVID-19

RESULTADOS DE LA SEGUNDA RONDA DE

RECOPILACIÓN DE DATOS

Population Council Guatemala

18 de agosto de 2020 


\section{Antecedentes}

- Según la Organización Panamericana de la Salud, la pandemia por la COVID-19 agudiza los factores de riesgo que históricamente han afectado la salud de los pueblos indígenas, incluyendo la falta de acceso a los servicios de salud, la desnutrición y el derecho insuficiente al agua y servicios básicos.

- Las condiciones de exclusión preexistentes generaran impactos desproporcionados a los municipios con mayoría de población indígena en el país.

- Para evaluar la situación en las comunidades indígenas, realizamos encuestas telefónicas con informantes clave en dos rondas (14-18 de abril y 21-26 julio).

- 10 municipalidades indígenas

- 144 entrevistas recolectadas en cada ronda 


\section{Resumen: Recopilación de datos de la Ronda 2}

- Grupos de informantes clave seleccionados por la trayectoria de trabajo del Population Council con comunidades indígenas por medio del program Abriendo Oportunidades.

- 108 informantes involucrados en la ronda 1 y 36 nuevos informantes incorporados en ronda 2 para una muestra total de 144 participantes

- Edad promedio: 38 años (rango de edad 19-67)

- 97\% viviendo en el mismo lugar que la ronda 1

- 96\% dispuestos a participar en futuras rondas 


\section{Grupos de informantes clave y ubicación}

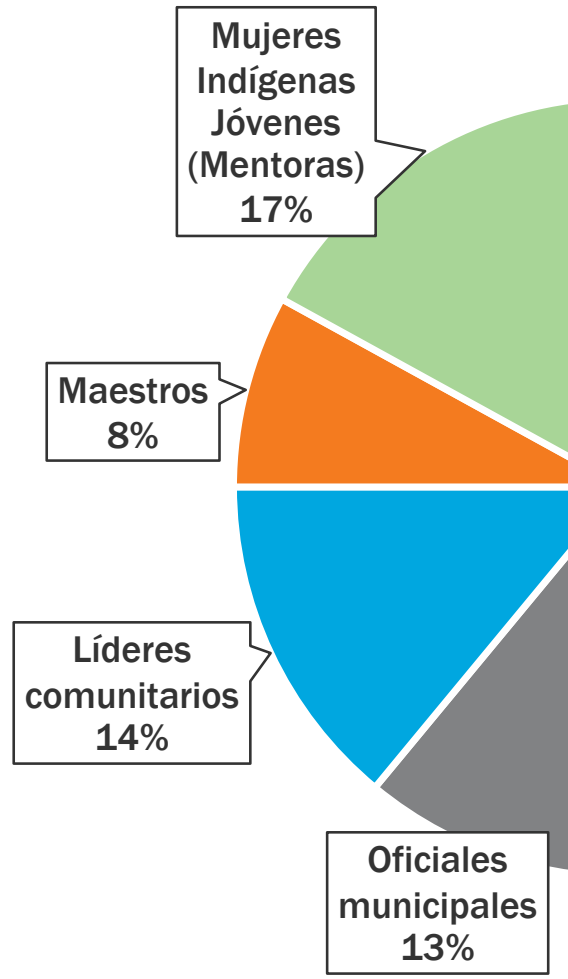

*Trabajadores de oficinas municipales (juventud, de la mujer, etc.)
$\mathrm{N}=144$

$23 \%$ hombres

$77 \%$ mujeres

Trabajadores de salud de primera línea

*Comadronas, promotores de salud comunitaria y

trabajadores de centros de salud

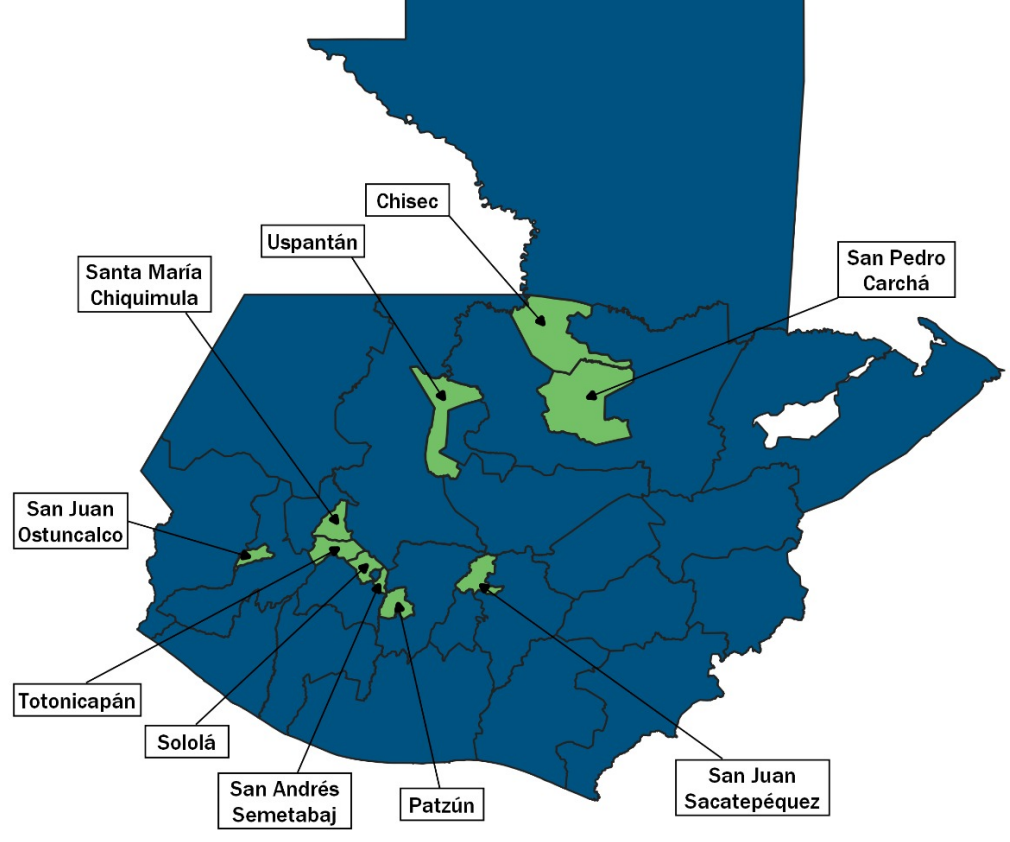




\section{¿Quién tiene un alto riesgo de enfermedad grave si está infectado de la COVID-19?}

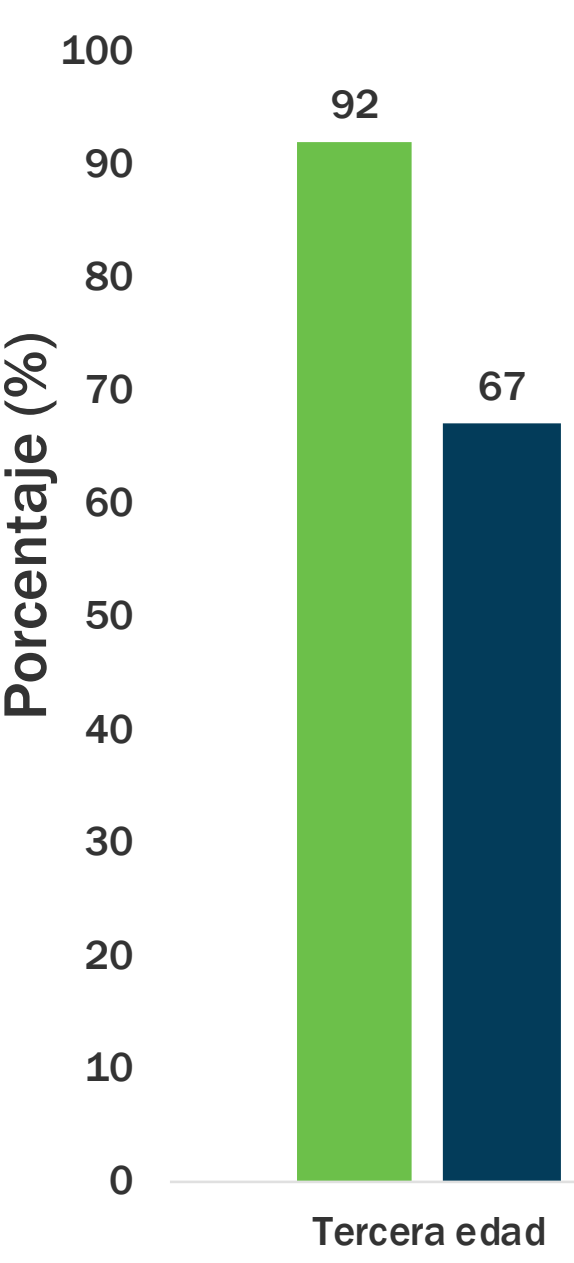

Los conocimientos sobre grupos en mayor riesgo son mixtos en ambas rondas, con una notable disminución en el riesgo para personas de la tercera edad.

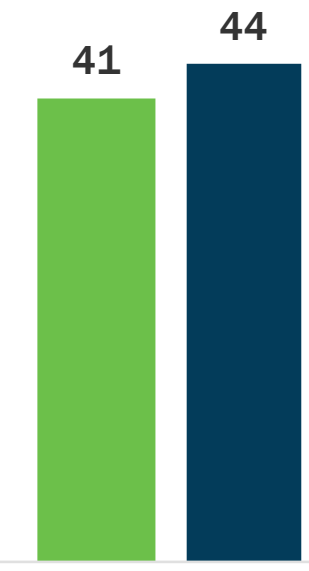

Personas con sistemas inmunitarios debilitados

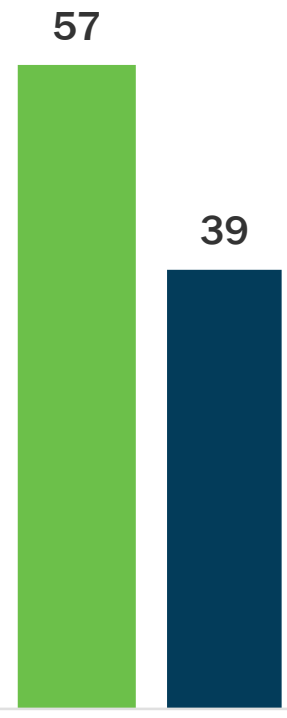

Niños
Ronda 1

- Ronda 2

28

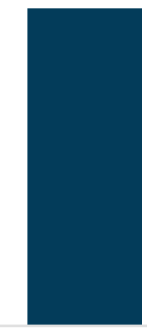

Todos 


\section{¿Cuáles son los síntomas de la COVID-19?}

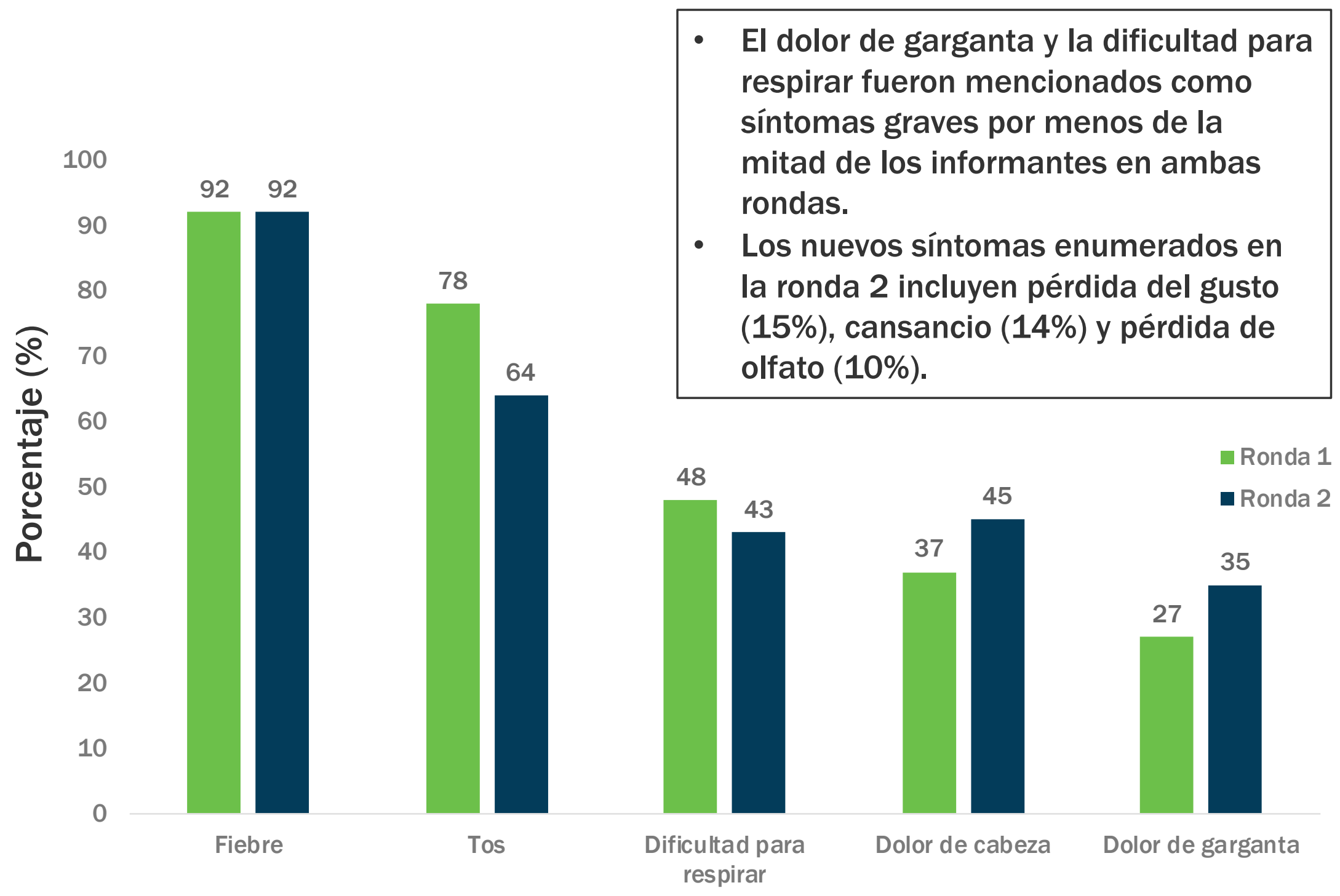




\section{Si tuviera síntomas de la COVID-19,}

¿qué haría?

100

90

80

$\frac{0}{0}$

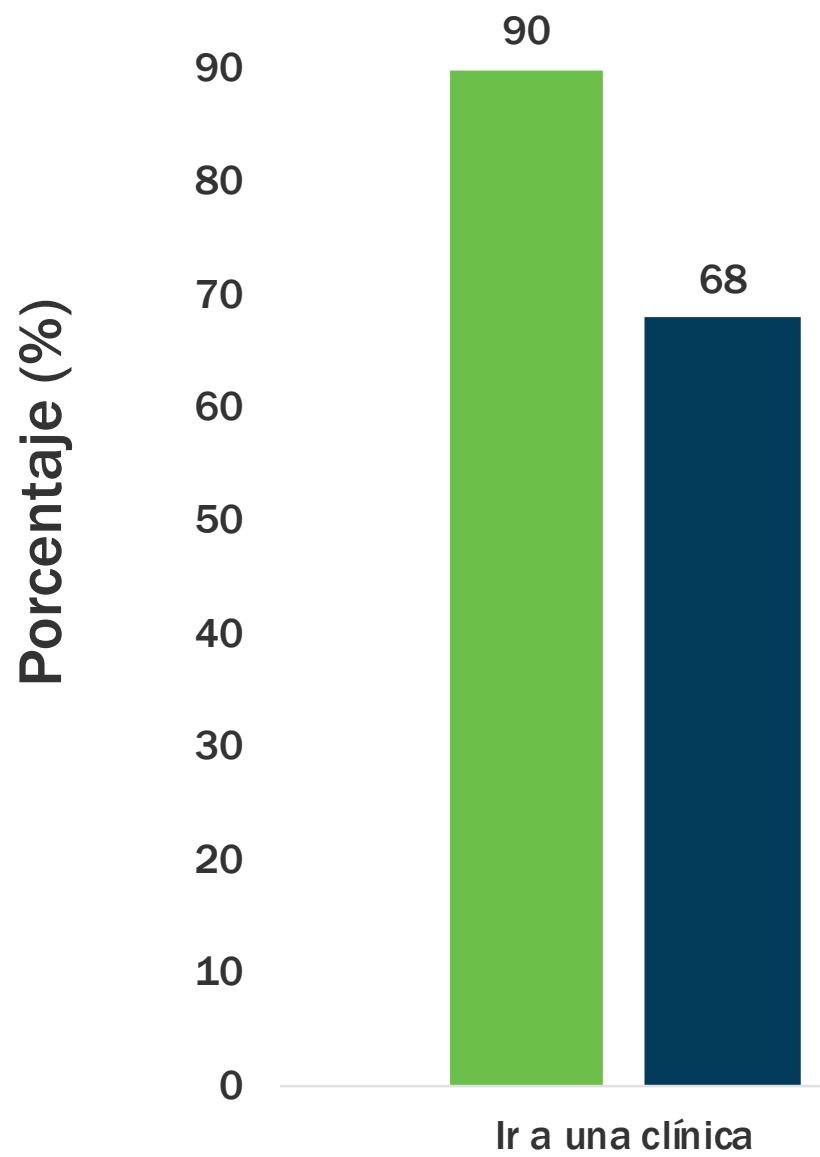

87

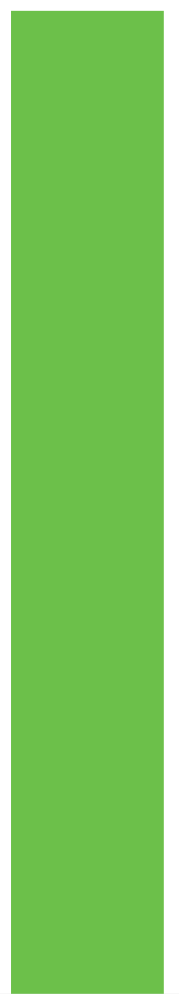

13

Hacerse la prueba de COVID-19

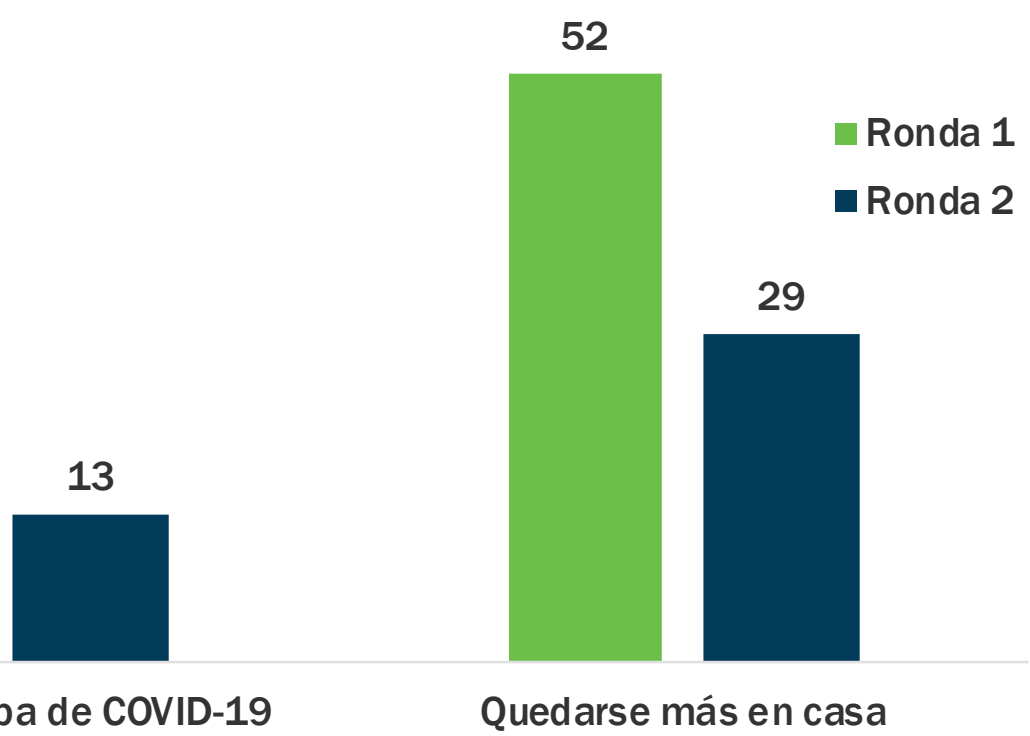

- Sólo el 37\% de los informantes que buscarían atención médica tendrían acceso a una clínica en su comunidad.

- El $54 \%$ tendría que viajar a la cabecera municipal más cercana. 


\section{Posibilidades de infectarse con la COVID-19}

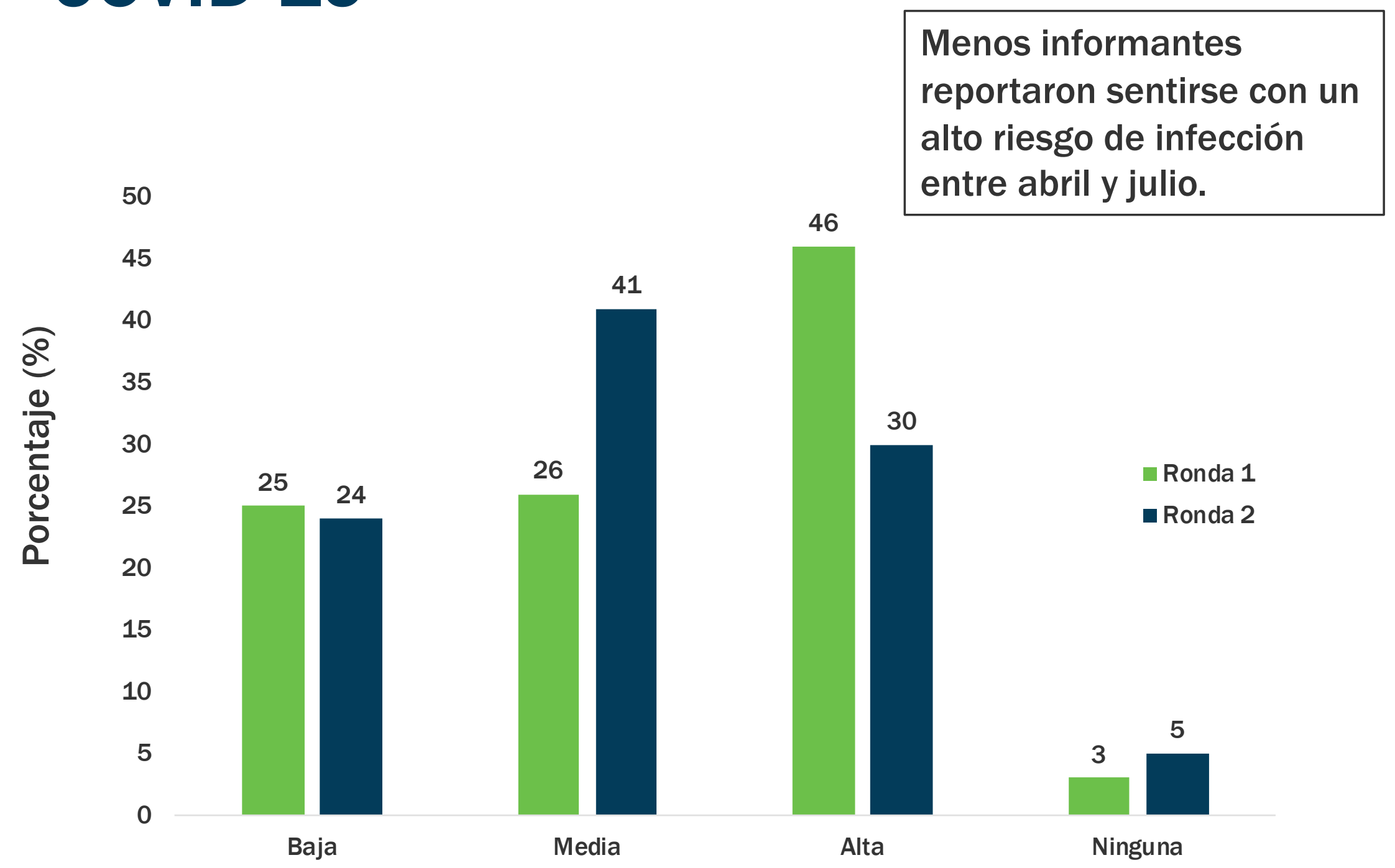




\section{¿De qué fuentes obtiene información sobre la COVID-19?}

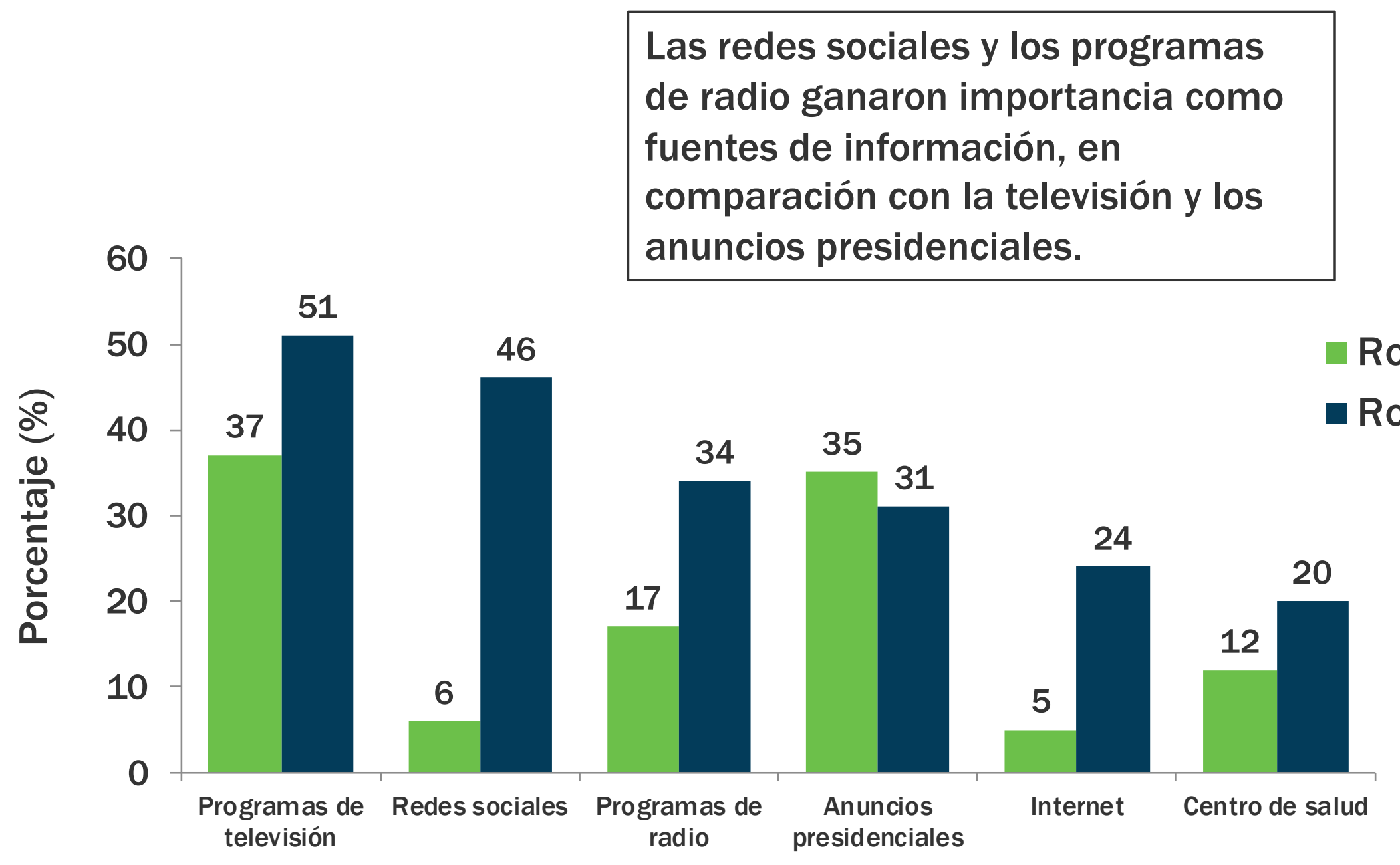




\section{Fuentes de las que se obtienen las mascarillas}

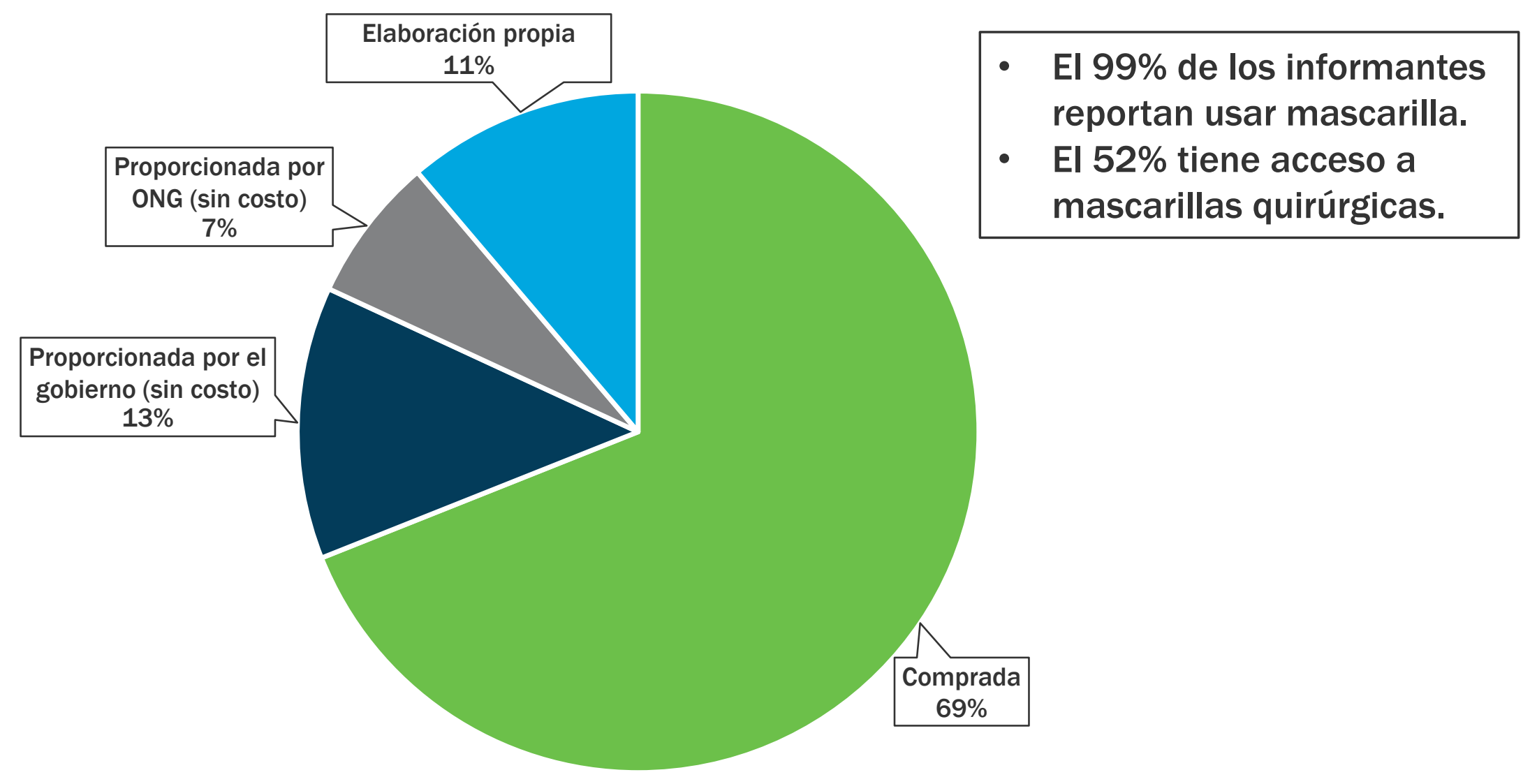




\section{Obstáculos al lavado de manos}

90

80

70

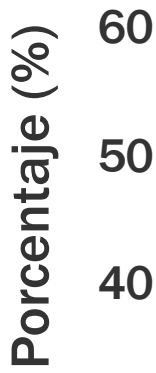

30

20

10

0

No pueden comprar agua/jabón
- El $66 \%$ de los encuestados dijo que las comunidades tienen acceso a estaciones de lavado de manos 0 desinfección.

- El $82 \%$ se lava las manos más de 7 veces al día.
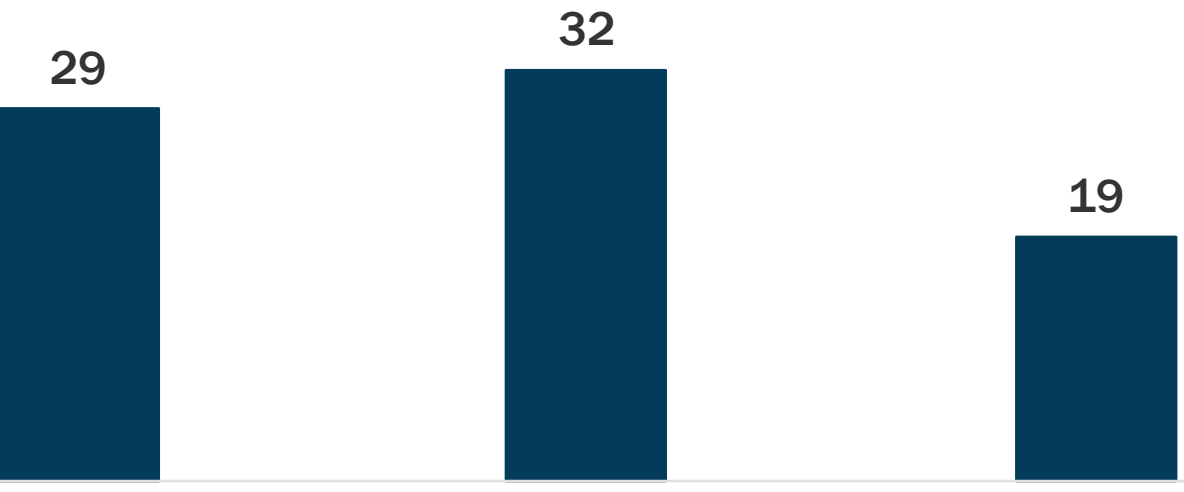

No pueden comprar desinfectante para manos

No tienen acceso a agua en su hogar 


\section{Efectos sociales y económicos del}

\section{COVID-19}

El 30\% de los participantes reportan saltarse las comidas o comer menos durante la crisis.

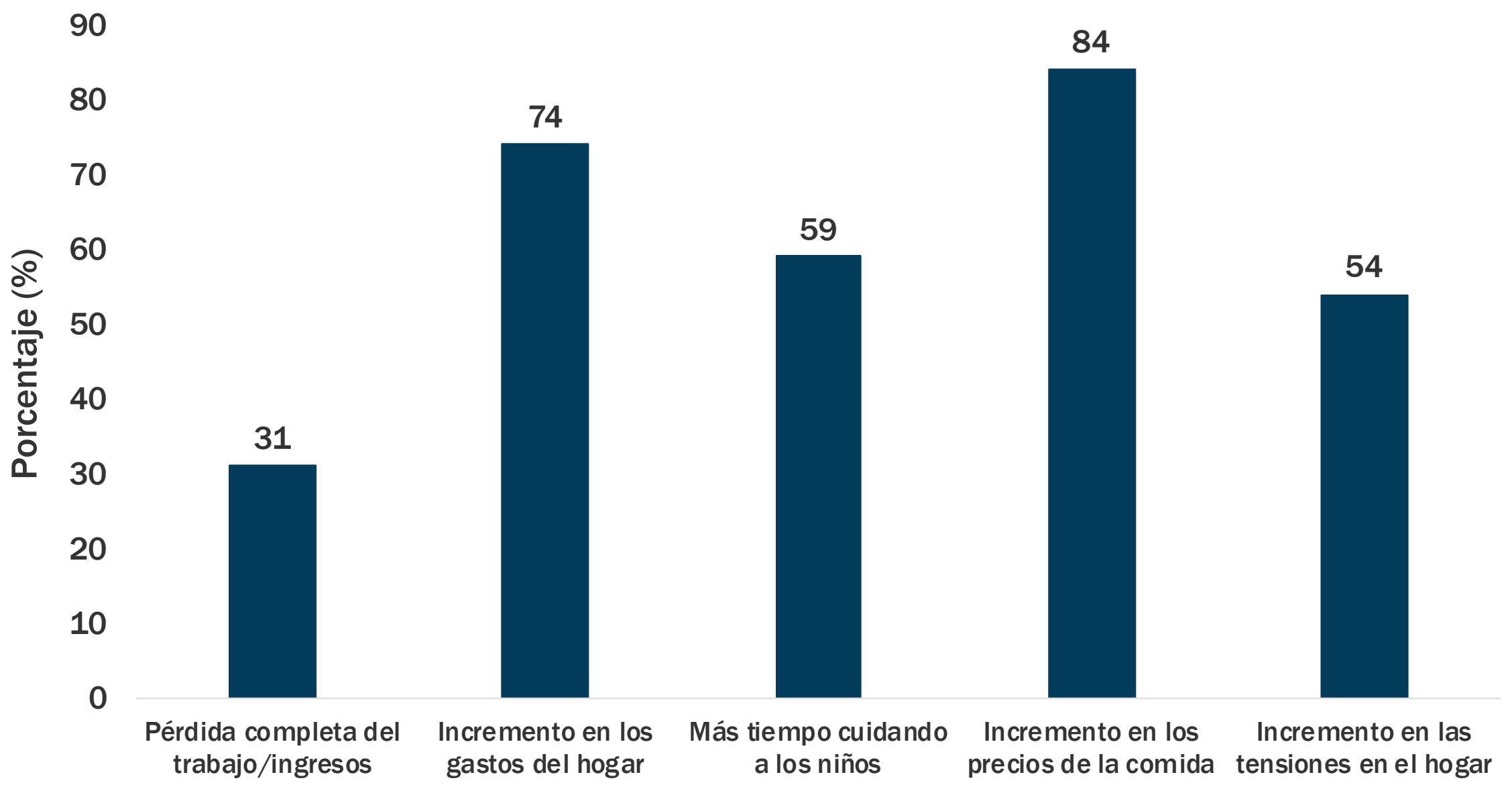




\section{Principales necesidades por las restricciones de prevención de la COVID-19}

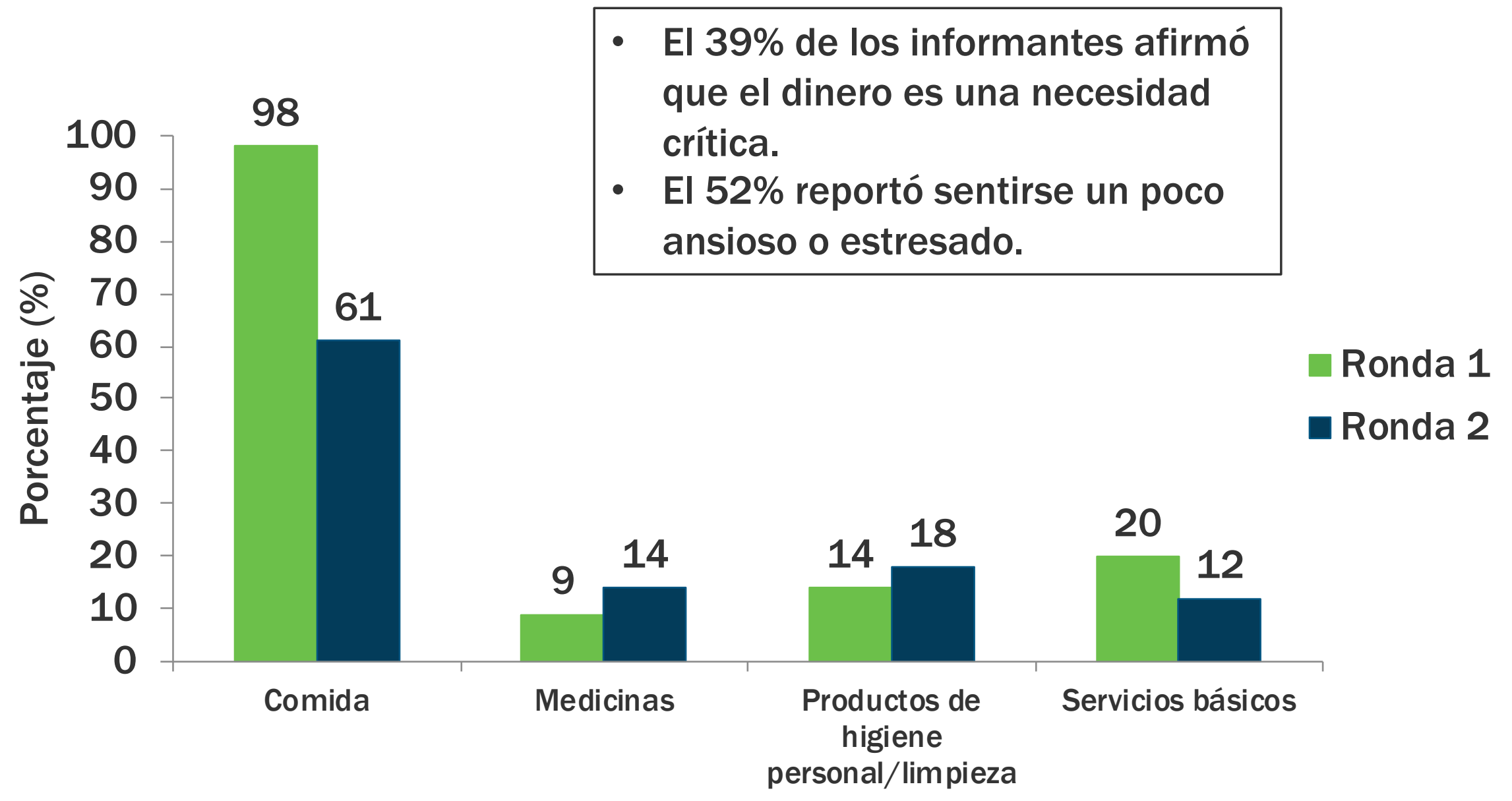




\section{Asistencia recibida (1)}

- $26 \%$ de los informantes han recibido cualquiera de los siguientes tipos de asistencia:

- 51\% dinero en efectivo/bono

- 32\% jabón/desinfectante

- 30\% víveres

- $22 \%$ Mascarillas

- Recibieron asistencia de las siguientes fuentes:

- $76 \%$ gobierno central

- $27 \%$ organizaciones no gubernamentales

- $8 \%$ gobierno municipal 


\section{Asistencia recibida (2)}

- El $72 \%$ de los informantes dijo saber a qué número llamar en caso de violencia en la comunidad

- El 91\% de los encuestados hombres ( $n=33)$ saben a qué número llamar en caso de violencia en comparación con el $66 \%$ de las mujeres encuestadas $(n=111)$.

- En el último mes, los informantes informan haber recibido visitas de cualquiera de las siguientes personas:

- $47 \%$ nadie

- 25\% líderes comunitarios

- $22 \%$ promotores de salud 


\section{Recomendaciones (1)}

1) Involucrar a los líderes y equipo de respuesta a nivel comunitario en acciones coordinadas para detectar los casos a tiempo, obtener acceso a pruebas, aislar casos positivos y rastrear y poner en cuarentena sus contactos.

2) Fortalecer la ruta de acción comunitaria de respuesta a la COVID-19 orientada a la atención de personas con síntomas graves y proporcionar guías sobre la derivación de pacientes al sistema nacional de salud cuando se requiera.

3) Apoyar a los trabajadores de salud de primera línea (promotores de la salud, enfermeras y comadronas) y líderes comunitarios con equipo de protección personal. 


\section{Recommendations (2)}

4) Difundir información específica en varios idiomas- a través del gobierno nacional y municipal-sobre los síntomas, las formas de transmisión y las características de las personas con un alto riesgo de enfermedad grave por la COVID-19.

5) Abordar los impactos económicos y sociales de la COVID-19, con especial atención a la inseguridad alimentaria, la experiencia de violencia en el hogar y la provisión de insumos que puedan aportar varios actores (gobierno, municipalidad, ONG, etc.) 


\title{
Información de contacto:
}

\author{
Angel del Valle, adelvalle@popcouncil.org \\ Corinne White, cwhite@popcouncil.org
}

Colaborando con los ministerios nacionales de salud y otros organismos gubernamentales en África subsahariana, Asia meridional y América Latina, científicos del Consejo de Población global y en el país están Ilevando a cabo investigaciones de salud pública y ciencias sociales COVID-19 para producir pruebas pertinentes y oportunas para apoyar a los responsables de la formulación de políticas en el control de la propagación del coronavirus, evaluar la eficacia de las medidas de prevención y mitigación, y evaluar los efectos a largo plazo para la salud, las redes sociales y económicas de la pandemia.

(C) 2020 The Population Council, Inc. 